\title{
Predictive value of combining the level of fibrinogen and antithrombin III for contrast-induced nephropathy in coronary artery disease patients undergoing percutaneous coronary intervention
}

\author{
YANHU SUN, DI ZHENG, QUAN ZHANG and WENHUA LI
}

Department of Cardiology, The Affiliated Hospital of Xuzhou Medical University, Xuzhou, Jiangsu 221002, P.R. China

Received September 17, 2019; Accepted July 1, 2020

DOI: 10.3892/br.2020.1333

\begin{abstract}
Contrast-induced nephropathy (CIN) is a common adverse event in the diagnosis and treatment of coronary intervention. The current study investigated the predictive effect of preoperative fibrinogen (FIB) combined with antithrombin III (AT-III) on CIN following percutaneous coronary intervention (PCI). A total of 394 patients who underwent PCI between October 2018 and May 2019 were selected for the present study. Pre-procedural FIB levels and AT-III activity were measured before PCI. CIN was defined as a $0.5 \mathrm{mg} / \mathrm{dl}$ or $25 \%$ increase in serum creatinine levels 48 to $72 \mathrm{~h}$ after exposure to a radiocontrast agent. Patients were classified into CIN and non-CIN groups. CIN occurred in 48 (12.2\%) patients. The serum FIB levels were significantly higher in patients who developed CIN compared with those who did not develop CIN. In addition, AT-III levels $\leq 89.5 \%$ were associated with higher rates of CIN. Logistical regression analysis showed that high FIB, and low AT-III and albumin levels were high-risk factors associated with CIN. For FIB, the area under the receiver operating characteristic curve (AUC) for predicting CIN was 0.653 . The optimal cut-off value was $3.48 \mathrm{~g} / \mathrm{l}$ with a sensitivity of $45.8 \%$ and a specificity of $79.7 \%$ [95\% confidence interval (CI): 0.603-0.701; $\mathrm{P}=0.0002)]$. For AT-III, the AUC was 0.711 , and the optimal cut-off value was $89.5 \%$, with a sensitivity of $81.3 \%$ and specificity of $58.2 \%$ (95\% CI: 0.659-0.758; $\mathrm{P}<0.0001)$. When combining FIB and AT-III, the AUC was 0.747. The optimal cut-off value was 0.090424 , with a diagnostic sensitivity of $93.8 \%$ and specificity of $46.6 \%$ (95\% CI: 0.697-0.792; P<0.0001). The results showed that FIB combined with AT-III resulted in improved predictive accuracy of CIN (FIB vs. AT-III, AUC=0.653 vs. 0.711, P=0.292; FIB
\end{abstract}

Correspondence to: Professor Wenhua Li, Department of Cardiology, The Affiliated Hospital of Xuzhou Medical University, 99 Huaihai West Road, Xuzhou, Jiangsu 221002, P.R. China

E-mail: xzwenhua0202@163.com

Key words: contrast-induced nephropathy, fibrinogen, antithrombin III, percutaneous coronary intervention vs. $\mathrm{FIB}+\mathrm{AT}-\mathrm{III}, \mathrm{AUC}=0.653$ vs. $0.747, \mathrm{P}=0.012$; AT-III vs. $\mathrm{FIB}+\mathrm{AT}-\mathrm{III}, \mathrm{AUC}=0.711$ vs. $0.747, \mathrm{P}=0.138)$. Pre-procedural levels of FIB, AT-III and albumin were independently associated with an increased risk of CIN. Furthermore, the results suggested that the combination of FIB and AT-III was a better predictor of CIN after PCI.

\section{Introduction}

Contrast-induced nephropathy (CIN) is a common adverse event observed in the diagnosis and treatment of coronary intervention (1). Renal function appears to deteriorate rapidly after the injection of an iodine contrast agent. CIN was first described in the 1950s in case reports of fatal acute renal failure that had occurred following intravenous pyelography in patients with renal disease arising from multiple myeloma (2). In the majority of cases, CIN is considered non-oliguric acute renal failure, with patients exhibiting asymptomatic transient decline in renal function. As a result, CIN may go undetected by clinicians who do not assess renal function in the days following contrast administration (3). The generally accepted definition of CIN is a $25 \%$ relative increase, or a $0.5 \mathrm{mg} / \mathrm{dl}$ ( $44 \mathrm{mmol} / \mathrm{l}$ ) absolute increase, in Scr levels within $48-72 \mathrm{~h}$ of contrast exposure, in the absence of an alternative explanation (4). The rise in Scr levels peaks after 3-5 days, and returns to baseline levels within 10-14 days (5). Risk factors associated with CIN include chronic kidney disease, contrast type and dose, diabetes mellitus, route of administration (intravenous vs. intra-arterial), congestive heart failure, age and use of nonsteroidal anti-inflammatory drugs (6). CIN prolongs hospitalization time and cost, and also worsens clinical symptoms, increases the incidence of adverse events, and carries with it a heavy economic burden for the patients and society (7). CIN is observed in $6-15.7 \%$ of patients who undergo coronary angiography in the setting of acute coronary syndrome (ACS) and contemporary management (8). At present, the pathogenesis of CIN is not fully understood, and there is no clear treatment. Therefore, early recognition of high-risk groups is particularly crucial for active prevention and treatment of CIN. Although several scoring systems used to predict the risk of CIN based on risk factors have been developed, identification of novel biomarkers may also assist in identifying at risk patients. 
Fibrinogen (FIB) or antithrombin III (AT-III) alone possess relatively little value for predicting the risk of CIN; however, the combination of the two factors has not been previously explored, to the best of our knowledge. Therefore, the current prospective study investigated the value of FIB combined with AT-III for predicting the risk of CIN after percutaneous coronary intervention (PCI).

\section{Patients and methods}

Patient population. All patients undergoing PCI admitted to The Affiliated Hospital of Xuzhou Medical University (Xuzhou, China) between October 2018 and May 2019 were prospectively enrolled in the present study. The exclusion criteria were: i) Administration of radio-contrast agents $48 \mathrm{~h}$ prior to the procedure or $72 \mathrm{~h}$ post-procedure; ii) lack of data on pre-procedural or post-procedural Scr levels; iii) administration of nonsteroidal nephrotoxic drugs $48 \mathrm{~h}$ before or $72 \mathrm{~h}$ post-PCI; iv) chronic kidney disease; v) patients with a malignant tumor, autoimmune disease, those who had undergone surgery recently, or patients with a history of trauma within the preceding month; vi) patients with a pulmonary thromboembolism, venous thromboembolism or a history of peripheral vascular disease; and vii) patients with infectious or inflammatory diseases. The contrast agent used was the low-osmotic and nonionic contrast agent iohexol, which was administered by injection (Yangtze River Pharmaceutical Group); the osmotic concentration was $\sim 800 \mathrm{mOsm} / \mathrm{Kg}$.

The final study population consisted of 394 patients (242 men and 152 women). The ages of the patients ranged from 29-95 years, with a median age of 66 years. In the CIN group, the age ranged from $42-95$ years, with a median age of 67 years, including 29 men and 19 women. In the non-CIN group, the age ranged from 29-85 years, with a median age of 66 years, and consisted of 213 men and 119 women. Patients were hydrated using normal saline at a rate of $1 \mathrm{ml} / \mathrm{kg} / \mathrm{h}$ at the start of the procedure or just before the procedure, and was continued for $12 \mathrm{~h}$ after the procedure ended. For emergency coronary interventional procedures, physiological $(0.9 \%)$ saline was administered intravenously at a rate of $1 \mathrm{ml} / \mathrm{kg} / \mathrm{h}$ for $12 \mathrm{~h}$ following contrast exposure. Coronary angiography and PCI were performed by interventional cardiologists, according to the standard clinical practice using the radial or femoral approach (9). All patients were administered aspirin (loading dose, $300 \mathrm{mg}$ ) and clopidogrel (loading dose, $300 \mathrm{mg}$ ) or ticagrelor (180 mg) upon presentation, and were subsequently administered a daily dose of aspirin (100 mg/day) and clopidogrel (75 mg/day) or ticagrelor $(90 \mathrm{mg} /$ day). The study protocol was performed in accordance with the Declaration of Helsinki (10) and was approved by the Ethics Committee of the Affiliated Hospital of Xuzhou Medical University. All patients provided written informed consent.

Diagnosis of CIN. CIN was defined as a Scr level that was $25 \%$ higher than before intravenous injection of contrast media, or an absolute value of $44.2 \mu \mathrm{mol} / 1(0.5 \mathrm{mg} / \mathrm{dl})$ within 48 to $72 \mathrm{~h}$, with other causes of renal damage excluded (11). According to the changes of Scr levels, patients were divided into a CIN group and non-CIN group.
Data collection. For all patients, the demographic characteristics, medical history, and variables related to the procedure were recorded. Routine laboratory data before and after the PCI procedures included routine tests of blood, liver and kidney function, as well as measurements of serum lipids, glucose, FIB and AT-III levels, amongst others.

All Scr levels were measured in a biochemical laboratory at the hospital using an Olympus AU2700 automatic biochemical analyzer (Olympus Corporation).

FIB and AT-III were measured using a Sysmex CA-7000 automatic coagulation analyzer and the analyzers original matching reagent (JEOL, Ltd.).

The estimated glomerular filtration rate was calculated using a modification of diet in renal disease equation for patients as follows: GFR $\left(\mathrm{ml} / \mathrm{min} / 1.73 \mathrm{~m}^{2}\right)=186 \mathrm{x} \mathrm{Scr}(\mathrm{mg} / \mathrm{dl})^{-1.154} \mathrm{x}$ age (years) $)^{-0.203} \mathrm{x}(0.742$ if female) (12).

Statistical analysis. All statistical analyses were performed using SPSS version 21.0 (IBM Corp.). For normally distributed data, continuous variables are shown as the mean \pm the standard deviation. Otherwise, the median and interquartile range (25-75\% values) are displayed, and non-parametric tests were used for analysis. Categorical variables are shown as the number and percentage (\%), and were compared using a $\chi^{2}$ test or Fisher's exact test. Comparison of parametric values between two groups was performed using an independent-samples Student's t-test. Comparisons of nonparametric values between two groups were compared using a Mann-Whitney U-test. Receiver operating characteristic (ROC) curve analysis was used to determine the optimal cut-off values of FIB and AT-III levels for detection of CIN using MedCalc version 19.06.6 (MedCalc Software byba). Multiple logistical regression analysis was used to identify independent risk factors of $\mathrm{CIN}$. $\mathrm{P}<0.05$ was considered to indicate a statistically significant difference.

\section{Results}

Baseline clinical characteristics and laboratory findings of the patients. The recruited cohort consisted of 394 patients receiving PCI with a mean age of $64.4 \pm 11.1$ years, and 242 patients $(61.4 \%)$ were men. A total of $48(12.2 \%)$ patients developed CIN. The univariate analysis of the clinicopathological characteristics and the baseline of the assessed parameters, including the conventional risk factors for CIN between the patients with and without CIN are shown in Table I. Distribution of sexes, hypertension and smoking status did not differ significantly between thew two groups. Additionally, there were no significant differences observed between the CIN and the non-CIN groups regarding the use of antihypertensive, antiplatelet or anti-ischemic, lipid-lowering drugs, or oral antidiabetic drugs. The average dose of contrast media in the CIN group was significantly larger than the dose administered in the non-CIN group $(\mathrm{P}=0.024)$.

Patients who developed CIN were likely to exhibit higher levels of FIB $(\mathrm{P}=0.001)$ and lower AT-III activity $(\mathrm{P}<0.001)$. Furthermore, these patients with CIN were likely to exhibit lower albumin levels $(\mathrm{P}<0.001)$. Patients who experienced a myocardial infarction were more likely to develop CIN $(\mathrm{P}=0.021)$. In the CIN group, the diuretic $(\mathrm{P}=0.015)$ and low molecular weight heparin usage $(\mathrm{P}=0.004)$ was significantly 
Table I. Baseline clinical characteristics of the CIN and non-CIN groups.

\begin{tabular}{|c|c|c|c|}
\hline Variables & Non-CIN, n=346 & $\mathrm{CIN}, \mathrm{n}=48$ & P-value \\
\hline Age, years & $64.17 \pm 10.95$ & $66.44 \pm 11.72$ & 0.182 \\
\hline Sex, male, n $(\%)$ & $213(61.6)$ & $29(60.4)$ & 0.879 \\
\hline Smoker, n (\%) & $114(32.9)$ & $20(41.7)$ & 0.234 \\
\hline hypertension, n (\%) & $199(57.5)$ & $27(56.3)$ & 0.868 \\
\hline Diabetes, n (\%) & $82(23.7)$ & $12(25.0)$ & 0.843 \\
\hline Body mass index, $\mathrm{kg} / \mathrm{m}^{2}$ & $25.88 \pm 3.34$ & $25.29 \pm 4.19$ & 0.268 \\
\hline Systolic blood pressure, $\mathrm{mmHg}$ & $133.14 \pm 19.47$ & $131.33 \pm 24.54$ & 0.626 \\
\hline Diastolic blood pressure, $\mathrm{mmHg}$ & $78.55 \pm 11.70$ & $79.42 \pm 14.86$ & 0.700 \\
\hline Contrast media, ml & $112.67 \pm 43.09$ & $127.50 \pm 37.27$ & $0.024^{\mathrm{a}}$ \\
\hline AMI, n (\%) & $108(31.2)$ & $23(47.9)$ & $0.021^{\mathrm{a}}$ \\
\hline \multicolumn{4}{|l|}{ Medication } \\
\hline$\beta$-blocker, n (\%) & $288(83.2)$ & $40(83.3)$ & 0.987 \\
\hline ACEI/ARB, n (\%) & $193(55.8)$ & $25(52.1)$ & 0.629 \\
\hline $\mathrm{CCB}, \mathrm{n}(\%)$ & $94(27.2)$ & $13(27.1)$ & 0.990 \\
\hline Diuretics, n (\%) & $118(34.1)$ & $25(52.1)$ & $0.015^{\mathrm{a}}$ \\
\hline Statin, n (\%) & $319(92.2)$ & $46(95.8)$ & 0.366 \\
\hline LMWH, n (\%) & $154(44.5)$ & $32(66.7)$ & $0.004^{\mathrm{b}}$ \\
\hline Nitrates, n (\%) & $221(63.9)$ & $31(64.6)$ & 0.923 \\
\hline
\end{tabular}

${ }^{\mathrm{a}} \mathrm{P}<0.05,{ }^{\mathrm{b}} \mathrm{P}<0.01$. CIN, contrast-induced nephropathy; AMI, acute myocardial infarction; ACEI/ARB, angiotensin converting enzyme inhibitors/angiotensin receptor blocker; CCB, calcium channel blockers; LMWH, low molecular weight heparin.

more common. The results of laboratory analysis are presented in Table II.

Multiple logistical regression analysis of risk factors for CIN. Multiple logistical regression analysis included the following factors: Acute myocardial infarction, albumin, contrast media volume, FIB, AT-III, and diuretic and low molecular weight heparin usage. Multivariate analyses indicated that albumin [odds ratio (OR), 0.893; 95\% confidence intervals (CI), 0.815-0.978; $\mathrm{P}=0.015)$, FIB (OR, 1.613; 95\% CI, 1.127-2.306; $\mathrm{P}=0.009)$ and AT-III (OR, 0.946; 95\% CI, 0.916-0.977; $\mathrm{P}=0.001$ ), were independent predictors for CIN (Table III).

Value of FIB and AT-III in predicting CIN. Regarding FIB, the area under the ROC curve (AUC) for predicting CIN was 0.653 . The optimal cut-off value was $3.48 \mathrm{~g} / \mathrm{l}$ with a sensitivity of $45.8 \%$ and specificity of $79.7 \%$ (95\% CI, 0.603-0.701; P=0.0002). For AT-III, the AUC was 0.711, and the optimal cut-off value was $89.5 \%$ with a sensitivity of $81.3 \%$ and specificity of $58.2 \%(95 \%$ CI, 0.659-0.758; $\mathrm{P}<0.001)$. FIB and AT-III were incorporated into the CIN risk factor regression analysis. The probability of the combination of FIB and AT-III predicting the occurrence of CIN was: Logit $(\mathrm{P})=2.059+0.600$ FIB-0.066 AT-III. The optimal cut-off value for the combination was calculated; when the predicted incidence of CIN was higher than 0.090424, patients were more likely to exhibit CIN following PCI. When combining FIB and AT-III, the AUC was 0.747. The optimal cut-off value was 0.090424 , with a diagnostic sensitivity of $93.8 \%$ and specificity of $46.6 \%$ (95\% CI, 0.697-0.792; $\mathrm{P}<0.001$ ). FIB combined with AT-III exhibited improved predictive accuracy in of CIN (FIB vs. AT-III, AUC $=0.653$ vs. $0.711, \mathrm{P}=0.292$;
FIB vs. FIB + AT-III, $\mathrm{AUC}=0.653$ vs. $0.747, \mathrm{P}=0.012$; AT-III vs. FIB + AT-III, AUC=0.711 vs. $0.747, \mathrm{P}=0.138)$. The results of ROC analysis are presented in Table IV and Fig. 1.

\section{Discussion}

The present study demonstrated that FIB, AT-III and albumin were independent predictors of CIN in patients undergoing PCI. Based on the results of the present study, the risk of CIN increased as the level of FIB increased or the activity of AT-III decreased. This study also suggested that the combination of FIB and AT-III was a better predictor of CIN after PCI.

With the increasing number of interventional procedures available, more patients will inevitably be assessed using iodine contrast agents as interventional procedures require its use, and these contrast reagents are associated with an increased risk of acute kidney injury (13). CIN results in a rapid decline of kidney function after administration of iodinated contrast material (14). $\mathrm{CIN}$ is one of the most common causes of hospitalization in patients with acquired kidney injury, and is known to increase the rates of morbidity and mortality, increase the costs of health care, as well as prolong the duration of hospitalization (15). At present, there are no specific treatment options for CIN (16). Therefore, early identification and intervention of patients with a high risk of experiencing CIN clinical events, is of great significance for improving patient outcomes.

FIB is a serum glycoprotein synthesized by hepatocytes. It is a major plasma coagulation factor and serves an important role in the inflammatory process (17). Increased levels of FIB result in an increase in blood viscosity, leading to shear stress damage in endothelial cells (18). Studies have shown that 
Table II. Laboratory results of the CIN and non-CIN groups.

A, Pre-operative

\begin{tabular}{|c|c|c|c|}
\hline Variables & Non-CIN, $n=348^{a}$ & $\mathrm{CIN}, \mathrm{n}=49^{\mathrm{a}}$ & P-value \\
\hline Triglyceride, mmol/l & $1.35(1.01-1.91)$ & $1.26(0.95-1.52)$ & 0.204 \\
\hline Total cholesterol, mmol/1 & $4.43 \pm 1.18$ & $4.28 \pm 1.07$ & 0.44 \\
\hline High-density lipoprotein, mmol/l & $1.12(0.95-1.32)$ & $1.13(0.98-1.36)$ & 0.72 \\
\hline Low-density lipoprotein, $\mathrm{mmol} / 1$ & $2.70 \pm 1.00$ & $2.59 \pm 0.79$ & 0.563 \\
\hline Albumin, g/l & $42.79 \pm 4.47$ & $39.33 \pm 3.93$ & $<0.001^{\mathrm{b}}$ \\
\hline Glucose, $\mathrm{mmol} / \mathrm{l}$ & $6.71 \pm 2.58$ & $7.15 \pm 3.02$ & 0.301 \\
\hline Fibrinogen, g/l & $2.90(2.42-3.35)$ & $3.23(2.80-3.80)$ & $0.001^{\mathrm{b}}$ \\
\hline Antithrombin III, \% & $91.54 \pm 11.73$ & $83.65 \pm 10.93$ & $<0.001^{\mathrm{b}}$ \\
\hline NLR & $2.99(2.05-5.03)$ & $3.29(2.41-5.85)$ & 0.305 \\
\hline PLR & $139.00(105.63-179.00)$ & $146.32(112.31-191.67)$ & 0.163 \\
\hline Serum creatinine, $\mu \mathrm{mol} / \mathrm{l}$ & $67.00(57.00-78.25)$ & $67.00(54.00-75.50)$ & 0.299 \\
\hline Cystatin C, mg/l & $0.78(0.71-0.91)$ & $0.81(0.71-0.88)$ & 0.985 \\
\hline Uric acid, $\mu \mathrm{mol} / 1$ & $306.50(248.00-365.00)$ & $296.50(258.00-349.75)$ & 0.513 \\
\hline $\mathrm{eGFR}, \mathrm{ml} / \mathrm{min}$ & $101.84 \pm 24.19$ & $107.04 \pm 34.23$ & 0.313 \\
\hline
\end{tabular}

B, Post-operative

\begin{tabular}{lccc}
\hline Serum creatinine, $\mu \mathrm{mol} / \mathrm{l}$ & $67.00(57.00-78.00)$ & $87.00(73.75-102.5)$ & $<0.001^{\mathrm{b}}$ \\
Cystatin C, $\mathrm{mg} / \mathrm{l}$ & $0.74(0.67-0.86)$ & $0.84(0.74-1.08)$ & $<0.001^{\mathrm{b}}$ \\
Uric acid, $\mu \mathrm{mol} / \mathrm{l}$ & $280.00(222.75-353.25)$ & $332.00(268.00-411.50)$ & 0.001 \\
$\mathrm{eGFR}, \mathrm{ml} / \mathrm{min}$ & $101.05 \pm 25.98$ & $75.54 \pm 25.04$ & $<0.001^{\mathrm{b}}$ \\
\hline
\end{tabular}

${ }^{a}$ Data are presented as the mean \pm standard deviation or the median and interquartile range $(25-75 \%)$. ${ }^{b} \mathrm{P} \leq 0.001$. CIN, contrast-induced nephropathy; NLR, neutrophil-to-lymphocyte ratio; PLR, platelet-to-lymphocyte ratio; eGFR, estimated glomerular filtration rate.

Table III. Multiple logistical regression analysis of risk factors for CIN.

\begin{tabular}{lcccccc}
\hline Variables & B & SE & Wald-value & P-value & Odds ratio & 95\% confidence intervals \\
\hline Albumin & -0.114 & 0.047 & 5.958 & $0.015^{\mathrm{a}}$ & 0.893 & $0.815-0.978$ \\
AT-III & -0.055 & 0.016 & 11.332 & $0.001^{\mathrm{b}}$ & 0.946 & $0.916-0.977$ \\
FIB & 0.478 & 0.183 & 6.848 & $0.009^{\mathrm{b}}$ & 1.613 & $1.127-2.306$ \\
Contrast media & 0.006 & 0.004 & 2.238 & 0.135 & 1.006 & $0.998-1.015$ \\
AMI & 0.223 & 0.391 & 0.327 & 0.568 & 1.250 & $0.581-2.690$ \\
Diuretic & -0.168 & 0.358 & 0.220 & 0.639 & 0.845 & $0.419-1.705$ \\
LMWH & -0.553 & 0.379 & 2.134 & 0.144 & 0.575 & $0.274-1.208$ \\
\hline
\end{tabular}

${ }^{\mathrm{a}} \mathrm{P}<0.05,{ }^{\mathrm{b}} \mathrm{P}<0.01$. CIN, contrast-induced nephropathy; B, regression coefficient; SE, standard error of the mean; AT-III, antithrombin III; FIB, fibrinogen; AMI, acute myocardial infarction; LMWH, low molecular weight heparin.

FIB in tissues is significantly elevated in acute kidney injury caused by ischemia-reperfusion (19-21). FIB causes severe renal dysfunction by increasing renal fibrosis (22).

Numerous previous studies have shown that serum FIB levels are associated with cardiovascular risk factors or cardiovascular events (23-26). Furthermore, FIB is associated with acute kidney injury (27), and studies have shown that FIB levels in tissues are significantly elevated in ischemia-induced acute renal injury caused by ischemia-reperfusion (22). FIB is an importantregulatorof coagulation, inflammation, woundhealing and angiogenesis, and functions by interacting with blood cells, endothelial cells and other types of cells (28). Additionally, FIB is an acute-phase protein and a marker of inflammation, as it is induced by cytokines, such as interleukin-6 $(29,30)$; thus, its expression is increased during inflammation. Inflammation is one of the primary mechanisms involved in the pathogenesis of CIN (31). Neutrophil-to-lymphocyte ratio (NLR) and platelet-to-lymphocyte ratio (PLR) are common inflammatory 
Table IV. Predictive values of FIB and AT-III for CIN based on the receiver operating characteristic curves.

\begin{tabular}{lcccccc}
\hline Variables & AUC & P-value & $95 \%$ CI & Sensitivity $(\%)$ & Specificity $(\%)$ & Cut-off value \\
\hline FIB & 0.653 & $0.0002^{\mathrm{a}}$ & $0.603-0.701$ & 45.8 & 79.7 & $3.48 \mathrm{~g} / \mathrm{l}$ \\
AT-III & 0.711 & $<0.0001^{\mathrm{a}}$ & $0.659-0.758$ & 81.3 & 58.2 & $89.5 \%$ \\
FIB + AT-III & 0.747 & $<0.0001^{\mathrm{a}}$ & $0.697-0.792$ & 93.8 & 46.6 & 0.090424
\end{tabular}

${ }^{\mathrm{a}} \mathrm{P}<0.001$. FIB, fibrinogen; AT-III, antithrombin III; AUC, area under the curve; CI, confidence interval; AUC, area under the curve.

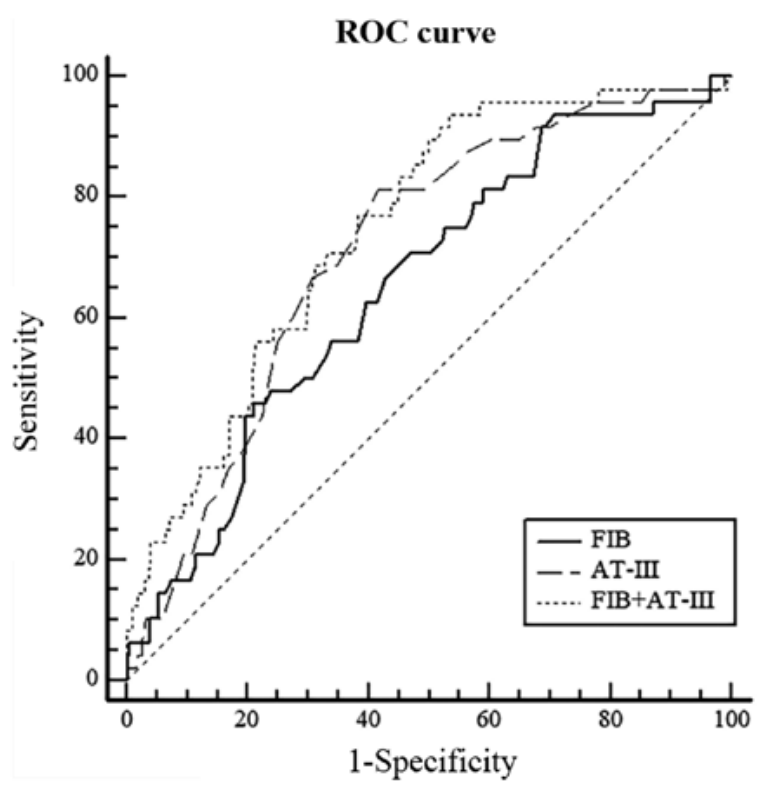

Figure 1. ROC curves for predicting contrast-induced nephropathy. ROC, receiver operating characteristic curve. FIB, fibrinogen; AT-III, antithrombin III.

markers. However, this difference was not significant. In addition, the increased levels of FIB may have increased blood viscosity, leading to insufficient perfusion of the kidney, and thus may increase the risk of CIN.

AT-III is the most important type of antithrombin, and its activity is often used as a laboratory indicator for judging anticoagulant levels and detection of thrombotic diseases (32). AT-III not only exhibited a strong anticoagulant effect, but also a strong anti-inflammatory effect. Thus AT-III levels may serve as a prognostic biomarker in neonatal sepsis, where a marked decrease in the levels of AT-III levels in septic neonates is observed (33). The decrease in the levels of AT-III is predictive of a poor outcome in sepsis (34). Furthermore, several studies have shown that AT-III exhibits a protective effect on contrast CIN (35). Previously, a study showed that patients with low levels of AT-III activity presented a high risk of developing acute kidney injury after cardiac surgery. Insufficiency of endogenous AT-III exacerbated the renal ischemia/reperfusion injury in rats (36). However, administration of exogenous AT-III may exhibit a protective effect against CIN through its anti-inflammatory and antioxidant effects, as well as by improving renal blood flow (35). The levels of AT-III were associated with the severity of coronary artery stenosis (37). Previous studies suggested that in patients with coronary artery disease, AT-III activity was closely associated with a hypercoagulable state or thrombosis. Additionally, the decrease in AT-III activity may indicate acute coronary events, and the extent of the decrease in activity level was positively correlated with the degree of coronary stenosis $(25,38-40)$.

However, to the best of our knowledge, there are no studies that have explored the value of combining FBI and AT-III to predict CIN. In the present study, it was shown that the combination of these two biomarkers exhibited improved predictive value for CIN. Additionally, a higher level of FIB was positively associated with the risk of CIN. In contrast to FIB, AT-III activity was a protective factor against CIN.

The present study has several limitations. First, the follow-up assessment of renal function was 1-3 days after PCI; therefore, a later increase in Scr levels in certain patients who did exhibit deteriorated renal function within $48 \mathrm{~h}$ of the procedure may have been missed. This oversight may result in a slight underestimation of CIN. Second, the number of enrolled patients was relatively small, particularly regarding patients with low AT-III activity levels, and all the patients recruited were from a single health care institution, and thus, a selection bias during enrollment was inevitable. In future studies, larger cohorts from multiple centers should be used to confirm the validity of the results presented.

In conclusion, the present study demonstrated that $3.48 \mathrm{~g} / \mathrm{l}$ was the optimal cut-off value of preoperative FIB, and $89.5 \%$ was the optimal cut-off value of preoperative AT-III, which were effective predictors of CIN following PCI. Furthermore, the combination of FIB and AT-III was a better predictor of a high risk of CIN compared with FIB alone. These results may provide a simple and easy indicator for use in predicting the risk of CIN.

\section{Acknowledgements}

Not applicable.

\section{Funding}

No funding was received.

\section{Availability of data and materials}

The datasets used and/or analyzed during the present study are available from the corresponding author on reasonable request.

\section{Authors' contributions}

YS designed the study and drafted the manuscript. DZ collected the data. QZ analyzed the data. WL designed the 
study, analyzed data and edited the manuscript. All authors read and approved the final manuscript.

\section{Ethics approval and consent to participate}

The study protocol was performed in accordance with the Declaration of Helsinki and was approved by the Ethics Committee of the Affiliated Hospital of Xuzhou Medical University (Xuzhou, China). All patients provided written informed consent.

\section{Patient consent for publication}

All patients signed informed consent approved by the Institutional Review Board of the Affiliated Hospital of Xuzhou Medical University.

\section{Competing interests}

The authors declare that they have no competing interests.

\section{References}

1. Mamoulakis C, Tsarouhas K, Fragkiadoulaki I, Heretis I, Wilks MF, Spandidos DA, Tsitsimpikou C and Tsatsakis A: Contrast-induced nephropathy: Basic concepts, pathophysiological implications and prevention strategies. Pharmacol Ther 180 99-112, 2017.

2. Katzberg RW and Haller C: Contrast-induced nephrotoxicity: Clinical landscape. Kidney Int Suppl: S3-S7, 2006.

3. Andreucci M, Faga T, Pisani A, Sabbatini M, Russo D and Michael A: Prevention of contrast-induced nephropathy through a knowledge of its pathogenesis and risk factors ScientificWorldJournal 2014: 823169, 2014.

4. Morcos SK and Thomsen HS; European Society of Urogenital Radiology: European society of urogenital radiology guidelines on administering contrast media. Abdom Imaging 28: 187-190, 2003.

5. Andreucci M, Solomon R and Tasanarong A: Side effects of radiographic contrast media: Pathogenesis, risk factors, and prevention. Biomed Res Int 2014: 741018, 2014.

6. Moos SI, van Vemde DN, Stoker J and Bipat S: Contrast induced nephropathy in patients undergoing intravenous (IV) contrast enhanced computed tomography (CECT) and the relationship with risk factors: A meta-analysis. Eur J Radiol 82: e387-e399, 2013.

7. McCullough PA, Adam A, Becker CR, Davidson C, Lameire N, Stacul F and Tumlin J; CIN Consensus Working Panel: Epidemiology and prognostic implications of contrast-induced nephropathy. Am J Cardiol 98: 5K-13K, 2006.

8. Guillon B, Ecarnot F, Marcucci C, Ducloux D, Chatot M, Badoz M, Bonnet B, Chopard R, Frey P, Meneveau N and Schiele F: Incidence, predictors, and impact on six-month mortality of three different definitions of contrast-induced acute kidney injury after coronary angiography. Am J Cardiol 121: 818-824, 2018

9. Gilchrist IC, Awuor SO, Davies RE and Ukaigwe AC: Controversies in complex percutaneous coronary intervention: Radial versus femoral. Expert Rev Cardiovasc Ther 15: 695-704, 2017.

10. General Assembly of the World Medical Association: World medical association declaration of Helsinki: Ethical principles for medical research involving human subjects. J Am Coll Dent 81: 14-18, 2014.

11. Rear R, Bell RM and Hausenloy DJ: Contrast-induced nephropathy following angiography and cardiac interventions. Heart 102 638-648, 2016.

12. Levey AS, Bosch JP, Lewis JB, Greene T, Rogers N and Roth D: A more accurate method to estimate glomerular filtration rate from serum creatinine: A new prediction equation. Modification of diet in renal disease study group. Ann Intern Med 130: 461-470, 1999
13. Muñoz de Bustillo Llorente E and de Miguel Balsa E: Radiological iodinated contrast-induced nephropathy. Rev Clin Esp 219: 403-410, 2019 (In English, Spanish).

14. Azzalini L, Spagnoli V and Ly HQ: Contrast-induced nephropathy: From pathophysiology to preventive strategies. Can J Cardiol 32: 247-255, 2016.

15. Gosling R and Iqbal J: Predicting contrast induced nephropathy in patients undergoing percutaneous coronary intervention. J Thorac Dis 11: 2672-2674, 2019.

16. Fähling M, Seeliger E, Patzak A and Persson PB: Understanding and preventing contrast-induced acute kidney injury. Nat Rev Nephrol 13: 169-180, 2017.

17. Fibrinogen Studies C, Danesh J, Lewington S, Thompson SG, Lowe GD, Collins R, Kostis JB, Wilson AC, Folsom AR, Wu K, et al: Plasma fibrinogen level and the risk of major cardiovascular diseases and nonvascular mortality: An individual participant meta-analysis. JAMA 294: 1799-1809, 2005.

18. Lowe GD, Fowkes FG, Dawes J, Donnan PT, Lennie SE and Housley E: Blood viscosity, fibrinogen, and activation of coagulation and leukocytes in peripheral arterial disease and the normal population in the Edinburgh artery study. Circulation 87: 1915-1920, 1993.

19. Druid H, Nilsson I, Rammer L and Skude G: Effect of anticoagulation on renal function and protein excretion in experimental acute ischemic renal failure. Ren Fail 21: 647-657, 1999.

20. Sörensen-Zender I, Rong S, Susnik N, Lange J, Gueler F, Degen JL, Melk A, Haller H and Schmitt R: Role of fibrinogen in acute ischemic kidney injury. Am J Physiol Renal Physiol 305: F777-F785, 2013

21. Huang MJ, Wei RB, Su TY, Wang Y, Li QP, Yang X, Lv XM and Chen XM: Impact of acute kidney injury on coagulation in adult minimal change nephropathy. Medicine (Baltimore) 95: e5366, 2016.

22. Krishnamoorthy A, Ajay AK, Hoffmann D, Kim TM, Ramirez V, Campanholle G, Bobadilla NA, Waikar SS and Vaidya VS: Fibrinogen $\beta$-derived $B \beta(15-42)$ peptide protects against kidney ischemia/reperfusion injury. Blood 118: 1934-1942, 2011.

23. Mayosi BM, Keavney BD, Watkins HC, Vickers MA and Green FR: Quantitative genetic study of plasma fibrinogen level and the- $455 \mathrm{G} / \mathrm{A}$ polymorphism of the beta-fibrinogen gene using novel family-based association methods. Circulation 102: 104 , 2000.

24. Carty CL, Cushman M, Jones D, Lange LA, Hindorff LA, Rice K, Jenny NS, Durda JP, Walston J, Carlson CS, et al: Associations between common fibrinogen gene polymorphisms and cardiovascular disease in older adults. The cardiovascular health study. Thromb Haemost 99: 388-395, 2008.

25. Thompson SG, Fechtrup C, Squire E, Heyse U, Breithardt G, van de Loo JC and Kienast J: Antithrombin III and fibrinogen as predictors of cardiac events in patients with angina pectoris. Arterioscl Throm Vas 16: 357-362, 1996.

26. De Luca G, Verdoia M, Cassetti E, Schaffer A, Cavallino C, Bolzani V; Novara Atherosclerosis Study Group (NAS): High fibrinogen level is an independent predictor of presence and extent of coronary artery disease among Italian population. J Thromb Thrombolysis 31: 458-463, 2011.

27. Docherty NG and Godson C: Fibrinogen as a damage-associated mitogenic signal for the renal fibroblast. Kidney Int 80: 1014-1016, 2011.

28. Laurens N, Koolwijk P and De Maat MP: Fibrin structure and wound healing. J Thromb Haemost 4: 932-939, 2006.

29. Kerr R, Stirling D and Ludlam CA: Interleukin 6 and haemostasis. Brit J Haematol 115: 3-12, 2001.

30. Szaba FM and Smiley ST: Roles for thrombin and fibrin(ogen) in cytokine/chemokine production and macrophage adhesion in vivo. Blood 99: 1053-1059, 2002.

31. Hossain MA, Costanzo E, Cosentino J, Patel C, Qaisar H, Singh V, Khan T, Cheng JS, Asif A and Vachharajani TJ: Contrast-induced nephropathy: Pathophysiology, risk factors, and prevention. Saudi J Kidney Dis Transpl 29: 1-9, 2018.

32. Meng R,LiZY, Ji X, Ding Y, Meng S and Wang X: Antithrombin III associated with fibrinogen predicts the risk of cerebral ischemic stroke. Clin Neurol Neurosur 113: 380-386, 2011.

33. Ersoy B, Nehir H, Altinoz S, Yilmaz O, Dundar PE and Aydogan A: Prognostic value of initial antithrombin levels in neonatal sepsis. Indian Pediatr 44: 581-584, 2007.

34. Samra N, AlGhwass M, Elgawhary S, Hassan M, Bekhit O, Mohamed W and Eid M: Serum level of antithrombin III (ATIII) could serve as a prognostic biomarker in neonatal sepsis. Fetal Pediatr Pathol 38: 290-298, 2019. 
35. Lu Z, Cheng D, Yin J, Wu R, Zhang G, Zhao Q, Wang N, Wang F and Liang M: Antithrombin III protects against contrast-induced nephropathy. EBioMedicine 17: 101-107, 2017.

36. Wang F, Zhang G, Lu Z, Geurts AM, Usa K, Jacob HJ, Cowley AW, Wang N and Liang M: Antithrombin III/SerpinC1 insufficiency exacerbates renal ischemia/reperfusion injury. Kidney Int 88: 796-803, 2015.

37. Lu J, Niu D, Zheng D, Zhang Q and Li W: Predictive value of combining the level of lipoprotein-associated phospholipase A2 and antithrombin III for acute coronary syndrome risk. Biomed Rep 9: 517-522, 2018.
38. Lipets EN and Ataullakhanov FI: Global assays of hemostasis in the diagnostics of hypercoagulation and evaluation of thrombosis risk. Thromb J 13: 4, 2015.

39. Brummel-Ziedins K, Undas A, Orfeo T, Gissel M, Butenas S, Zmudka K and Mann KG: Thrombin generation in acute coronary syndrome and stable coronary artery disease: Dependence on plasma factor composition. J Thromb Haemost 6: 104-110, 2008.

40. Hong X, Shan PR, Hu L, Huang ZQ, Wu GJ, Xiao FY and Huang WJ: Relationship between antithrombin-III value with acute coronary syndrome and preprocedural TIMI flow grade. Zhonghua Yi Xue Za Zhi 92: 831-834, 2012 (In Chinese). 